\title{
PROJECTIVE STOCHASTIC EQUATIONS AND NONLINEAR LONG MEMORY
}

\author{
IEVA GRUBLYTE் $* * *$ AND \\ DONATAS SURGAILIS, ${ }^{* * * *}$ Vilnius University
}

\begin{abstract}
A projective moving average $\left\{X_{t}, t \in \mathbb{Z}\right\}$ is a Bernoulli shift written as a backward martingale transform of the innovation sequence. We introduce a new class of nonlinear stochastic equations for projective moving averages, termed projective equations, involving a (nonlinear) kernel $Q$ and a linear combination of projections of $X_{t}$ on 'intermediate' lagged innovation subspaces with given coefficients $\alpha_{i}$ and $\beta_{i, j}$. The class of such equations includes usual moving average processes and the Volterra series of the LARCH model. Solvability of projective equations is obtained using a recursive equality for projections of the solution $X_{t}$. We show that, under certain conditions on $Q, \alpha_{i}$, and $\beta_{i, j}$, this solution exhibits covariance and distributional long memory, with fractional Brownian motion as the limit of the corresponding partial sums process.
\end{abstract}

Keywords: Projective stochastic equation; long memory; LARCH model; Bernoulli shift; invariance principle

2010 Mathematics Subject Classification: Primary 60G10

Secondary $60 \mathrm{~F} 17 ; 60 \mathrm{H} 25$

\section{Introduction}

A discrete-time second-order stationary process $\left\{X_{t}, t \in \mathbb{Z}\right\}$ is called a long memory process if its covariance $\gamma(k)=\operatorname{cov}\left(X_{0}, X_{k}\right)$ decays slowly with the lag in such a way that its absolute series diverges: $\sum_{k=1}^{\infty}|\gamma(k)|=\infty$. In the converse case when $\sum_{k=1}^{\infty}|\gamma(k)|<\infty$ and $\sum_{k=-\infty}^{\infty} \gamma(k) \neq 0$ the process $\left\{X_{t}\right\}$ is called a short memory process. Long memory processes have different properties from short memory (in particular, independent and identically distributed (i.i.d.)) processes. Long memory processes have been found to arise in a variety of physical and social sciences. See, e.g. the monographs [2], [8], [10], and the references therein.

Probably the most important model of long memory processes is the linear, or moving average, process

$$
X_{t}=\sum_{s \leq t} b_{t-s} \zeta_{s}, \quad t \in \mathbb{Z}
$$

where $\left\{\zeta_{s}, s \in \mathbb{Z}\right\}$ is a standardized i.i.d. sequence, and the moving average coefficients $b_{j}$ decay slowly so that $\sum_{j=0}^{\infty}\left|b_{j}\right|=\infty$ and $\sum_{j=0}^{\infty} b_{j}^{2}<\infty$. The last condition guarantees that the series in (1) converges in mean square and satisfies $\mathbb{E} X_{t}=0$ and $\mathbb{E} X_{t}^{2}=\sum_{j=0}^{\infty} b_{j}^{2}<\infty$. In the literature it is often assumed that the coefficients regularly decay as

$$
b_{j} \sim \kappa j^{d-1} \quad \text { as } j \rightarrow \infty \text { for } \kappa>0 \text { and } d \in\left(0, \frac{1}{2}\right) .
$$

\footnotetext{
Received 27 March 2013; revision received 9 December 2013.

* Postal address: Institute of Mathematics and Informatics, Akademijos 4, LT-08663 Vilnius, Lithuania.

** Email address: ieva.grublyte@mii.vu.lt

*** Email address: donatas.surgailis@mii.vu.lt
} 
Condition (2) guarantees that

$$
\gamma(k)=\sum_{j=0}^{\infty} b_{j} b_{k+j} \sim \kappa^{2} B(d, 1-2 d) k^{2 d-1} \quad \text { as } k \rightarrow \infty
$$

and, hence, $\sum_{k=1}^{\infty}|\gamma(k)|=\infty$. The parameter $d$ in (2) is called the long memory parameter of $\left\{X_{t}\right\}$. A particular case of the linear process in (1)-(2) is the parametric class of fractional integrated, autoregressive, moving average, $\operatorname{ARFIMA}(p, d, q)$, in which case $d \in(0,1 / 2)$ is the order of fractional integration. An important property of the linear process in (1)-(2) is the fact that its (normalized) partial sums process $S_{n}(\tau):=\sum_{j=1}^{[n \tau]} X_{j}, \tau \geq 0$, tends to a fractional Brownian motion (see [4]), namely,

$$
n^{-d-1 / 2} S_{n}(\tau) \rightarrow{ }_{D[0,1]} \sigma(d) B_{H}(\tau),
$$

where $H=d+\frac{1}{2}$ is the Hurst parameter, $\sigma(d)^{2}:=\kappa^{2} B(d, 1-2 d) / d(1+2 d)>0$ and ' $\rightarrow D[0,1]$ ' denotes the weak convergence of random processes in the Skorokhod space $D[0,1]$.

On the other hand, the linear model (1) has its drawbacks and sometimes is not capable of incorporating empirical features ('stylized facts') of some observed time series. The 'stylized facts' may include typical asymmetries, clusterings, and other nonlinearities which are often observed in financial data, together with long memory.

In the present paper we introduce a new class of nonlinear processes which generalize the linear model in (1)-(2) and enjoy similar long memory properties to (3) and (4). These processes are defined through solutions of the so-called projective stochastic equations. Here the term 'projective' refers to the fact that these equations contain linear combinations of projections, or conditional expectations, of the $X_{t}$ on lagged innovation subspaces which enter the equation in a nonlinear way.

Let us explain the main idea of our construction. We call a projective moving average a random process $\left\{X_{t}\right\}$ of the form

$$
X_{t}=\sum_{s \leq t} g_{s, t} \zeta_{s}, \quad t \in \mathbb{Z}
$$

where $\left\{\zeta_{s}\right\}$ is a sequence of standardized i.i.d. random variables as in (1), $g_{t, t} \equiv g_{0}$ is a deterministic constant, and $g_{s, t}, s<t$, are random variables depending only on $\zeta_{s+1}, \ldots, \zeta_{t}$ such that

$$
g_{s, t}=g_{t-s}\left(\zeta_{s+1}, \ldots, \zeta_{t}\right), \quad s<t,
$$

where $g_{j}: \mathbb{R}^{j} \rightarrow \mathbb{R}, j=1,2, \ldots$, are nonrandom functions satisfying

$$
\sum_{s \leq t} \mathbb{E} g_{s, t}^{2}=\sum_{s \leq 0} \mathbb{E} g_{-s}^{2}\left(\zeta_{s+1}, \ldots, \zeta_{0}\right)<\infty .
$$

It follows easily that, under condition (7), the series in (5) converges in mean square; define a stationary process with zero mean and finite variance $\mathbb{E} X_{t}^{2}=\sum_{s \leq t} \mathbb{E} g_{s, t}^{2}$. A natural question to ask is how to choose the 'coefficients' $g_{s, t}$ in (6) so that they depend on $X_{t}$ and behave like (2) when $j=t-s \rightarrow \infty$.

A particularly simple choice for the $g_{s, t}$ to achieve the above goals is

$$
g_{s, t}=b_{t-s} Q\left(\mathbb{E}_{[s+1, t]} X_{t}\right), \quad s \leq t,
$$


where the $b_{j}$ are as in (2), $Q: \mathbb{R} \rightarrow \mathbb{R}$ is a given deterministic kernel, and $\mathbb{E}_{[s+1, t]} X_{t}:=$ $\mathbb{E}\left[X_{t} \mid \zeta_{v}, s+1 \leq v \leq t\right]$ is the projection of $X_{t}$ onto the subspace of $L^{2}$ generated by the innovations $\zeta_{v}, s+1 \leq v \leq t$ (the conditional expectation). The corresponding projective stochastic equation has the form

$$
X_{t}=\sum_{s \leq t} b_{t-s} Q\left(\mathbb{E}_{[s+1, t]} X_{t}\right) \zeta_{s}
$$

Note that, when $s \rightarrow-\infty, \mathbb{E}_{[s+1, t]} X_{t} \rightarrow X_{t}$ by a general property of the conditional expectation and then $g_{s, t} \sim b_{t-s} Q\left(X_{t}\right)$ if $Q$ is continuous. This means that the $g_{s, t}$ in (8) feature both the long memory in (2) and the dependence on the 'current' value $X_{t}$ through $Q\left(X_{t}\right)$. In particular, for $Q(x)=\max (0, x)$, the behavior of $g_{s, t}$ in (8) strongly depends on the sign of $X_{t}$ and the trajectory of (9) appears very asymmetric (see the top diagram of Figure 3 in Section 5).

Let us briefly describe the remaining sections. Section 2 contains basic definitions and properties of projective processes. In Section 3 we introduce a general class of projective stochastic equations, (9) being a particular case. We obtain sufficient conditions for solvability of these equations, and a recurrent formula for the computation of the 'coefficients' $g_{s, t}$ (Theorem 1). In Sections 4 and 5 we present some examples and simulated trajectories and histograms of projective equations. It turns out that the LARCH model studied in [12] and elsewhere is a particular case of projective equations corresponding to the linear kernel $Q(x)$ (Section 4). Some modifications of projective equations are discussed in Section 6. In Section 7 we deal with long memory properties of stationary solutions of stochastic projective equations. We show that, under some additional conditions, these solutions have long memory properties similar to (3) and (4).

Finally, we note that 'nonlinear long memory' is a general term and that other time series models different from ours for such behavior were proposed in the literature. Among them, probably the most studied class are subordinated processes of the form $\left\{Q\left(X_{t}\right)\right\}$, where $\left\{X_{t}\right\}$ is a Gaussian or linear long memory process and $Q: \mathbb{R} \rightarrow \mathbb{R}$ is a nonlinear function. See [10], [16], and [22] for a detailed discussion. A related class of Gaussian subordinated stochastic volatility models is studied in [20]. Doukhan et al. [7] discussed a class of long memory Bernoulli shifts. Baillie and Kapetanios [1] considered the fractionally integrated process with nonlinear autoregressive innovations. A general invariance principle for fractionally integrated models with weakly dependent innovations satisfying the projective dependence condition of $\mathrm{Wu}$ [23] is established in [25]. See also [24] and Remark 5 below.

We expect that the results of this paper can be extended in several directions, e.g. projective equations with initial conditions, continuous-time processes, random field setups, and infinite variance processes. For applications, a major challenge is the estimation of the 'parameters' of projective equations. We plan to study some of these issues in the future.

\section{Projective processes and their properties}

Let $\left\{\zeta_{t}, t \in \mathbb{Z}\right\}$ be a sequence of i.i.d. random variables with $\mathbb{E} \zeta_{0}=0$ and $\mathbb{E} \zeta_{0}^{2}=1$. For any integers $s \leq t$, we define $\mathcal{F}_{[s, t]}:=\sigma\left\{\zeta_{u}: u \in[s, t]\right\}$ to be the sigma-algebra generated by $\zeta_{u}, u \in[s, t], \mathcal{F}_{(-\infty, t]}:=\sigma\left\{\zeta_{u}: u \leq t\right\}$, and $\mathcal{F}:=\sigma\left\{\zeta_{u}: u \in \mathbb{Z}\right\}$. For $s>t$, we define $\mathcal{F}_{[s, t]}:=\{\varnothing, \Omega\}$ as the trivial sigma-algebra. Let $L_{[s, t]}^{2}, L_{(-\infty, t]}^{2}$, and $L^{2}$ be the spaces of all square-integrable random variables $\xi$ measurable with respect to $\mathcal{F}_{[s, t]}, \mathcal{F}_{(-\infty, t]}$, and $\mathcal{F}$, respectively. For any $s, t \in \mathbb{Z}$, let

$$
\mathbb{E}_{[s, t]}[\xi]:=\mathbb{E}\left[\xi \mid \mathcal{F}_{[s, t]}\right], \quad \xi \in L^{2},
$$


be the conditional expectation. Then $\xi \mapsto \mathbb{E}_{[s, t]}[\xi]$ is a bounded linear operator in $L^{2}$; moreover, $\mathbb{E}_{[s, t]}, s, t \in \mathbb{Z}$, is a projection family satisfying $\mathbb{E}_{\left[s_{2}, t_{2}\right]} \mathbb{E}_{\left[s_{1}, t_{1}\right]}=\mathbb{E}_{\left[s_{2}, t_{2}\right] \cap\left[s_{1}, t_{1}\right]}$ for any intervals $\left[s_{1}, t_{1}\right],\left[s_{2}, t_{2}\right] \subset \mathbb{Z}$. From the definition of the conditional expectation, it follows that if $g_{u}: \mathbb{R} \rightarrow \mathbb{R}, u \in \mathbb{Z}$, are arbitrary measurable functions with $\mathbb{E} g_{u}^{2}\left(\zeta_{u}\right)<\infty,\left[s_{2}, t_{2}\right] \subset \mathbb{Z}$ is a given interval, and $\xi=\prod_{u \in\left[s_{2}, t_{2}\right]} g_{u}\left(\zeta_{u}\right)$ is a product of independent random variables, then, for any interval $\left[s_{1}, t_{1}\right] \subset \mathbb{Z}$,

$$
\mathbb{E}_{\left[s_{1}, t_{1}\right]} \prod_{u \in\left[s_{2}, t_{2}\right]} g_{u}\left(\zeta_{u}\right)=\prod_{u \in\left[s_{1}, t_{1}\right] \cap\left[s_{2}, t_{2}\right]} g_{u}\left(\zeta_{u}\right) \prod_{v \in\left[s_{2}, t_{2}\right] \backslash\left[s_{1}, t_{1}\right]} \mathbb{E}\left[g_{v}\left(\zeta_{v}\right)\right] .
$$

In particular, if $\mathbb{E} g_{u}\left(\zeta_{u}\right)=0, u \in \mathbb{Z}$, then

$$
\mathbb{E}_{\left[s_{1}, t_{1}\right]} \prod_{u \in\left[s_{2}, t_{2}\right]} g_{u}\left(\zeta_{u}\right)= \begin{cases}\prod_{u \in\left[s_{1}, t_{1}\right]} g_{u}\left(\zeta_{u}\right), & {\left[s_{2}, t_{2}\right] \subset\left[s_{1}, t_{1}\right],} \\ 0, & {\left[s_{2}, t_{2}\right] \not \subset\left[s_{1}, t_{1}\right] .}\end{cases}
$$

Any random variable $Y_{t} \in L_{(-\infty, t]}^{2}$ can be expanded as the orthogonal series $Y_{t}=\mathbb{E} Y_{t}+$ $\sum_{s \leq t} P_{s, t} Y_{t}$, where $P_{s, t} Y_{t}:=\left(\mathbb{E}_{[s, t]}^{\infty, t]}-\mathbb{E}_{[s+1, t]}\right) Y_{t}$. Note that $\left\{P_{s, t} Y_{t}, \mathcal{F}_{s, t}, s \leq t\right\}$ is a backward martingale difference sequence and $\mathbb{E} Y_{t}^{2}=\left(\mathbb{E} Y_{t}\right)^{2}+\sum_{s \leq t} \mathbb{E}\left(P_{s, t} Y_{t}\right)^{2}$.

Definition 1. A projective process is a random sequence $\left\{Y_{t} \in L_{(-\infty, t]}^{2}, t \in \mathbb{Z}\right\}$ of the form

$$
Y_{t}=\mathbb{E} Y_{t}+\sum_{s \leq t} g_{s, t} \zeta_{s}
$$

where the $g_{s, t}$ are random variables satisfying the following conditions:

(i) $g_{s, t}$ is $\mathcal{F}_{[s+1, t]}$-measurable for all $s, t \in \mathbb{Z}, s<t$; $g_{t, t}$ is a deterministic number;

(ii) $\sum_{s \leq t} \mathbb{E} g_{s, t}^{2}<\infty$ for all $t \in \mathbb{Z}$.

In other words, a projective process has the property that the projections $\mathbb{E}_{[s, t]} Y_{t}=\mathbb{E} Y_{t}+$ $\sum_{i=s}^{t} P_{i, t} Y_{t}=\mathbb{E} Y_{t}+\sum_{i=s}^{t} \zeta_{i} g_{i, t}, s \leq t$, form a backward martingale transform with respect to the nondecreasing family $\left\{\mathcal{F}_{[s, t]}, s \leq t\right\}$ of sigma-algebras for each fixed $t \in \mathbb{Z}$. A consequence of the last fact is the following moment inequality which is an easy consequence of Rosenthal's inequality (see [14, p. 24]). See also [10, Lemma 2.5.3].

Proposition 1. Let $\left\{Y_{t}\right\}$ be a projective process of the form (10). Assume that $\mu_{p}:=\mathbb{E}\left|\zeta_{0}\right|^{p}<$ $\infty$ and $\sum_{s \leq t}\left(\mathbb{E}\left|g_{s, t}\right|^{p}\right)^{2 / p}<\infty$ for some $p \geq 2$. Then $\mathbb{E}\left|Y_{t}\right|^{p}<\infty$. Moreover, there exists a constant $C_{p}<\infty$ depending only on $p$ and such that

$$
\mathbb{E}\left|Y_{t}\right|^{p} \leq C_{p}\left(\left|\mathbb{E} Y_{t}\right|^{p}+\mu_{p}\left(\sum_{s \leq t}\left(\mathbb{E}\left|g_{s, t}\right|^{p}\right)^{2 / p}\right)^{p / 2}\right)
$$

Definition 2. A projective moving average is a projective process of the form (10) such that the mean $\mathbb{E} Y_{t}=\mu$ is constant, and there exist a number $g_{0} \in \mathbb{R}$ and nonrandom measurable functions $g_{j}: \mathbb{R}^{j} \rightarrow \mathbb{R}, j=1,2, \ldots$, such that

$$
g_{s, t}=g_{t-s}\left(\zeta_{s+1}, \ldots, \zeta_{t}\right) \quad \text { almost surely for any } s \leq t, s, t \in \mathbb{Z} .
$$


By definition, a projective moving average is a stationary Bernoulli shift (see [6, p. 21]), i.e.

$$
Y_{t}=\mu+\sum_{s \leq t} \zeta_{s} g_{t-s}\left(\zeta_{s+1}, \ldots, \zeta_{t}\right)
$$

with mean $\mu$ and covariance

$$
\begin{aligned}
\operatorname{cov}\left(Y_{s}, Y_{t}\right) & =\sum_{u \leq s} \mathbb{E}\left[g_{s-u}\left(\zeta_{u+1}, \ldots, \zeta_{s}\right) g_{t-u}\left(\zeta_{u+1}, \ldots, \zeta_{t}\right)\right] \\
& =\sum_{u \leq 0} \mathbb{E}\left[g_{-u}\left(\zeta_{u+1}, \ldots, \zeta_{0}\right) g_{t-s-u}\left(\zeta_{u+1}, \ldots, \zeta_{t-s-u}\right)\right], \quad s \leq t
\end{aligned}
$$

These facts together with the ergodicity of Bernoulli shifts (implied by a general result in [21, Theorem 3.5.8]) are summarized in the following corollary.

Corollary 1. A projective moving average is a strictly stationary and ergodic stationary process with finite variance and covariance given in (12).

Remark 1. If the coefficients $g_{s, t}$ are nonrandom, a projective moving average is a linear process $Y_{t}=\mu+\sum_{s \leq t} g_{t-s} \zeta_{s}, t \in \mathbb{Z}$.

Proposition 2. Let $\left\{Y_{t}\right\}$ be a projective process of the form (10), and let $\left\{a_{j}, j \geq 0\right\}$ be a deterministic sequence with $\sum_{j=0}^{\infty}\left|a_{j}\right|<\infty$ and $\sum_{j=0}^{\infty}\left|a_{j}\right|\left|\mathbb{E} Y_{t-j}\right|<\infty$. Then $\left\{u_{t}:=\right.$ $\left.\sum_{j=0}^{\infty} a_{j} Y_{t-j}, t \in \mathbb{Z}\right\}$ is a projective process $u_{t}=\mathbb{E} u_{t}+\sum_{s \leq t} \zeta_{s} G_{s, t}$ with $\mathbb{E} u_{t}=$ $\sum_{j=0}^{\infty} a_{j} \mathbb{E} Y_{t-j}$ and coefficients $G_{s, t}:=\sum_{j=0}^{t-s} a_{j} g_{s, t-j}$.

Proof. The proof easily follows by the Cauchy-Schwarz inequality and is thus omitted.

Proposition 3. If $\left\{Y_{t}\right\}$ is a projective process of the form (10) then, for any $s \leq t$,

$$
\mathbb{E}_{[s, t]} Y_{t}=\mathbb{E} Y_{t}+\sum_{s \leq u \leq t} \zeta_{u} g_{u, t}, \quad P_{s, t} Y_{t}=\left(\mathbb{E}_{[s, t]}-\mathbb{E}_{[s+1, t]}\right) Y_{t}=\zeta_{s} g_{s, t} .
$$

Representation (10) is unique: if (10) and $Y_{t}=\sum_{s \leq t} g_{s, t}^{\prime} \zeta_{s}$ are two representations, with $g_{s, t}^{\prime}$ satisfying conditions ( $i$ ) and (ii) of Definition 1, then $g_{s, t}^{\prime}=g_{s, t}$ for all $s \leq t$.

Proof. Equation (13) is immediate by definition of the projective process. From (13), it follows that $\zeta_{s} g_{s, t}^{\prime \prime}=0$, where $g_{s, t}^{\prime \prime}:=g_{s, t}-g_{s, t}^{\prime}$ is independent of $\zeta_{s}$. The relation $\mathbb{E} \zeta_{s}^{2}=1$ implies that $\mathbb{P}\left(\left|\zeta_{s}\right|^{2}>\varepsilon\right)>0$ for all small enough $\varepsilon>0$. Hence, $0=\mathbb{P}\left(\left|\zeta_{s} g_{s, t}^{\prime \prime}\right|>\varepsilon\right) \geq$ $\mathbb{P}\left(\left|\zeta_{s}\right|>\sqrt{\varepsilon},\left|g_{s, t}^{\prime \prime}\right|>\sqrt{\varepsilon}\right)=\mathbb{P}\left(\left|\zeta_{s}\right|>\sqrt{\varepsilon}\right) \mathbb{P}\left(\left|g_{s, t}^{\prime \prime}\right|>\sqrt{\varepsilon}\right)$, implying that $\mathbb{P}\left(\left|g_{s, t}^{\prime \prime}\right|>\sqrt{\varepsilon}\right)=0$ for any $\varepsilon>0$.

The following invariance principle is due to Dedecker and Merlevède [5, Corollary 3]; see also [23, Theorem 3 (i)].

Proposition 4. Let $\left\{Y_{t}\right\}$ be a projective moving average of the form (10) such that $\mu=0$ and

$$
\Omega(2):=\sum_{t=0}^{\infty}\left\|g_{0, t}\right\|<\infty,
$$

where $\|\xi\|=\mathbb{E}^{1 / 2}\left[\xi^{2}\right], \xi \in L^{2}$. Then

$$
n^{-1 / 2} \sum_{t=1}^{[n \tau]} Y_{t} \rightarrow{ }_{D[0,1]} c_{Y} B(\tau)
$$

where $B$ is a standard Brownian motion and $c_{Y}^{2}:=\left\|\sum_{t=0}^{\infty} g_{0, t}\right\|^{2}=\sum_{t \in \mathbb{Z}} \mathbb{E}\left[Y_{0} Y_{t}\right]$. 


\section{Projective stochastic equations}

Let $Q_{s, t}=Q_{s, t}\left(x_{u, v}, s<u \leq v \leq t\right), s, t \in \mathbb{Z}, s<t$, be some given measurable deterministic functions depending on $(t-s)(t-s+1) / 2, s<t$, real variables $x_{u, v}$, and let $\mu_{t}, Q_{t, t}, t \in \mathbb{Z}$, be some given constants. A projective stochastic equation has the form

$$
X_{t}=\mu_{t}+\sum_{s \leq t} \zeta_{s} Q_{s, t}\left(\mathbb{E}_{[u, v]} X_{v}, s<u \leq v \leq t\right)
$$

Definition 3. By a solution of (16) we mean a projective process $\left\{X_{t}, t \in \mathbb{Z}\right\}$ satisfying

$$
\sum_{s \leq t} \mathbb{E}\left[Q_{s, t}^{2}\left(\mathbb{E}_{[u, v]} X_{v}, s<u \leq v \leq t\right)\right]<\infty
$$

and (16) for any $t \in \mathbb{Z}$.

Proposition 5. Assume that $\mu_{t}=\mu$ does not depend on $t \in \mathbb{R}$, that the functions $Q_{s, t}=$ $Q_{t-s}, s \leq t$, in (16) depend only on $t-s$, and that $\left\{X_{t}\right\}$ is a solution of (16). Then $\left\{X_{t}\right\}$ is a projective moving average of the form (11) with $\mathbb{E} X_{t}=\mu$ and $g_{n}: \mathbb{R}^{n} \rightarrow \mathbb{R}, n=0,1, \ldots$, defined recursively by

$$
g_{n}\left(x_{-n+1}, \ldots, x_{0}\right):=Q_{n}\left(\mu+\sum_{k=u}^{v} x_{k} g_{v-k}\left(x_{u+1}, \ldots, x_{v}\right),-n<u \leq v \leq 0\right), \quad n \geq 1,
$$

and $g_{0}:=Q_{0}$. Moreover, such a solution is unique.

Proof. From (16) and the uniqueness of (10) (Proposition 3), we have $X_{t}=\mu+\sum_{s \leq t} g_{s, t} \zeta_{s}$, where $g_{s, t}=Q_{t-s}\left(\mathbb{E}_{[u, v]} X_{v}, s<u \leq v \leq t\right)$. For $s=t$, this yields $g_{t, t}=Q_{0}=g_{0}$ for all $t \in \mathbb{Z}$ as in (17). Similarly, $g_{t-1, t}=Q_{1}\left(\mathbb{E}_{[t, t]} X_{t}\right)=Q_{1}\left(\mu+g_{0} \zeta_{t}\right)=g_{1}\left(\zeta_{t}\right)$, where $g_{1}$ is defined in (17). Assume by induction that

$$
g_{t-m, t}=g_{m}\left(\zeta_{t-m+1}, \ldots, \zeta_{t}\right) \text { for all } t \in \mathbb{Z},
$$

with $g_{m}$ defined in (17), holds for any $0 \leq m<n$ and some $n \geq 1$; we need to show that (18) holds for $m=n$, too. Using (18), (13), and (17), we obtain

$$
\begin{aligned}
g_{t-n, t} & =Q_{n}\left(\mathbb{E}_{[u, v]} X_{v}, t-n<u \leq v \leq t\right) \\
& =Q_{n}\left(\mu+\sum_{k=u}^{v} \zeta_{k} g_{v-k}\left(\zeta_{u+1}, \ldots, \zeta_{v}\right), t-n<u \leq v \leq t\right) \\
& =g_{n}\left(\zeta_{t-n+1}, \ldots, \zeta_{t}\right) .
\end{aligned}
$$

This proves the induction step $n-1 \rightarrow n$ and, hence, the proposition, since the uniqueness follows trivially.

Clearly, the choice of possible kernels $Q_{s, t}$ in (16) is very large. In this paper we focus on the following class of projective stochastic equations:

$$
X_{t}=\mu+\sum_{s \leq t} \zeta_{s} Q\left(\alpha_{t-s}+\sum_{s<u \leq t} \beta_{t-u, u-s}\left(\mathbb{E}_{[u, t]} X_{t}-\mathbb{E}_{[u+1, t]} X_{t}\right)\right)
$$


Here $\left\{\alpha_{i}, i \geq 0\right\}$ and $\left\{\beta_{i, j}, i \geq 0, j \geq 1\right\}$ are given arrays of real numbers, $\mu \in \mathbb{R}$ is a constant, and $Q=Q(x)$ is a measurable function of a single variable $x \in \mathbb{R}$. Two modifications of (19) are briefly discussed below; see (41) and (44). Particular cases of (19) are

$$
X_{t}=\sum_{s \leq t} \zeta_{s} Q\left(\alpha_{t-s}+\beta_{t-s} \mathbb{E}_{[s+1, t]} X_{t}\right)
$$

and

$$
X_{t}=\mu+\sum_{s \leq t} \zeta_{s} Q\left(\alpha_{t-s}+\sum_{s<u \leq t} \beta_{u-s}\left(\mathbb{E}_{[u, t]} X_{t}-\mathbb{E}_{[u+1, t]} X_{t}\right)\right),
$$

corresponding to $\beta_{i, j}=\beta_{i+j}$ and $\beta_{i, j}=\beta_{j}$, respectively.

Next, we study the solvability of the projective equation (19). We assume that $Q$ satisfies the following dominating bound: there exists a constant $c_{Q}>0$ such that

$$
|Q(x)| \leq c_{Q}|x| \text { for all } x \in \mathbb{R} \text {. }
$$

Define

$$
K_{Q}:=\sum_{i=0}^{\infty} \alpha_{i}^{2} \sum_{k=0}^{\infty} c_{Q}^{2 k+2} \sum_{j_{1}=1}^{\infty} \beta_{i, j_{1}}^{2} \cdots \sum_{j_{k}=1}^{\infty} \beta_{i+j_{1}+\cdots+j_{k-1}, j_{k}}^{2} .
$$

The main result of this section is the following theorem.

Theorem 1. (i) Assume that condition (22) holds and that

$$
K_{Q}<\infty
$$

Then there exists a unique solution $\left\{X_{t}\right\}$ of (19), which is written as a projective moving average of the form (10) with coefficients $g_{t-k, t}$ recursively defined as

$$
g_{t-k, t}:= \begin{cases}Q\left(\alpha_{k}+\sum_{i=0}^{k-1} \beta_{i, k-i} \zeta_{t-i} g_{t-i, t}\right), & k=1,2, \ldots \\ Q\left(\alpha_{k}\right), & k=0 .\end{cases}
$$

More explicitly,

$$
\begin{aligned}
X_{t}= & \mu+Q\left(\alpha_{0}\right) \zeta_{t}+Q\left(\alpha_{1}+\beta_{0,1} \zeta_{t} Q\left(\alpha_{0}\right)\right) \zeta_{t-1} \\
& +Q\left(\alpha_{2}+\beta_{0,2} \zeta_{t} Q\left(\alpha_{0}\right)+\beta_{1,1} \zeta_{t-1} Q\left(\alpha_{1}+\beta_{0,1} \zeta_{t} Q\left(\alpha_{0}\right)\right)\right) \zeta_{t-2}+\cdots
\end{aligned}
$$

(ii) In the case of the linear function $Q(x)=c_{Q} x$, condition (24) is also necessary for the existence of a solution to (19).

Proof. (i) Let us show that the $g_{k-t, t}$ as defined in (25) satisfy $\sum_{k=0}^{\infty} \mathbb{E} g_{t-k, t}^{2}<\infty$. From (22) and (25), we have the recurrent inequality

$$
\mathbb{E} g_{t-k, t}^{2} \leq c_{Q}^{2} \mathbb{E}\left(\alpha_{k}+\sum_{i=0}^{k-1} \beta_{i, k-i} \zeta_{t-i} g_{t-i, t}\right)^{2}=c_{Q}^{2}\left(\alpha_{k}^{2}+\sum_{i=0}^{k-1} \beta_{i, k-i}^{2} \mathbb{E} g_{t-i, t}^{2}\right)
$$


Iterating (26) we obtain

$$
\begin{aligned}
\mathbb{E}_{t-k, t}^{2} & \leq c_{Q}^{2}\left(\alpha_{k}^{2}+c_{Q}^{2} \sum_{i=0}^{k-1} \beta_{i, k-i}^{2}\left(\alpha_{i}^{2}+\sum_{j=0}^{i-1} \beta_{j, i-j}^{2} \mathbb{E} g_{t-j, t}^{2}\right)\right) \\
& =c_{Q}^{2} \alpha_{k}^{2}+c_{Q}^{4} \sum_{i=0}^{k-1} \alpha_{i}^{2} \beta_{i, k-i}^{2}+c_{Q}^{6} \sum_{i=0}^{k-1} \alpha_{i}^{2} \sum_{j_{1}=1}^{k-1-i} \beta_{i, j_{1}}^{2} \beta_{i+j_{1}, k-i-j_{1}}^{2}+\cdots
\end{aligned}
$$

and, hence,

$$
\begin{aligned}
\sum_{k=0}^{\infty} \mathbb{E} g_{t-k, t}^{2} & \leq c_{Q}^{2} \sum_{i=0}^{\infty} \alpha_{i}^{2}+c_{Q}^{4} \sum_{i=0}^{\infty} \alpha_{i}^{2} \sum_{j_{1}=1}^{\infty} \beta_{i, j_{1}}^{2}+c_{Q}^{6} \sum_{i=0}^{\infty} \alpha_{i}^{2} \sum_{j_{1}=1}^{\infty} \beta_{i, j_{1}}^{2} \sum_{j_{2}=1}^{\infty} \beta_{i+j_{1}, j_{2}}^{2}+\cdots \\
& =K_{Q} \\
& <\infty
\end{aligned}
$$

according to (24). Therefore, $X_{t}=\mu+\sum_{s \leq t} g_{s, t} \zeta_{s}$ is a well-defined projective moving average. The remaining statements about $X_{t}$ follow from Proposition 5.

(ii) Similarly to (26), and (28) in the case $Q(x)=c_{Q} x$ we obtain

$$
\mathbb{E} g_{t-k, t}^{2}=c_{Q}^{2} \mathbb{E}\left(\alpha_{k}+\sum_{i=0}^{k-1} \beta_{i, k-i} \zeta_{t-i} g_{t-i, t}\right)^{2}=c_{Q}^{2}\left(\alpha_{k}^{2}+\sum_{i=0}^{k-1} \beta_{i, k-i}^{2} \mathbb{E} g_{t-i, t}^{2}\right),
$$

and, hence, $\operatorname{var}\left(X_{t}\right)=\sum_{k=0}^{\infty} \mathbb{E} g_{t-k, t}^{2}=K_{Q}$. This proves (ii), completing the proof.

Remark 2. From recurrent relation (25), the $g_{t-k, t}$ can be expressed as functions of $\zeta_{t-k+1}$, $\ldots, \zeta_{t}$ via the so-called nested Volterra series (see the extended version of this paper available at http://arxiv.org/abs/1312.1938v1).

In the case of (20) and (21), condition (24) can be simplified; see below. Note that, for $A^{2}:=\sum_{i=0}^{\infty} \alpha_{i}^{2}=0$, the equations in (25) admit the trivial solution $g_{t-k, t}=0$ since $Q(0)=0$ by (22), leading to the constant process $X=\mu$ in (19).

Proposition 6. (i) Let $A^{2}>0, \beta_{i, j}=\beta_{i+j}, i \geq 0, j \geq 1$, and $B^{2}:=\sum_{i=0}^{\infty} \beta_{i}^{2}$. Then $K_{Q}<\infty$ is equivalent to $A^{2}<\infty$ and $B^{2}<\infty$.

(ii) Let $A^{2}>0, \beta_{i, j}=\beta_{j}, i \geq 0, j \geq 1$, and $B^{2}:=\sum_{i=1}^{\infty} \beta_{i}^{2}$. Then $K_{Q}<\infty$ is equivalent to $A^{2}<\infty$ and $c_{Q}^{2} B^{2}<1$. Moreover, $K_{Q}=c_{Q}^{2} A^{2} /\left(1-c_{Q}^{2} B^{2}\right)$.

Proof. (i) By definition,

$$
\begin{aligned}
K_{Q} & =\sum_{k=0}^{\infty} c_{Q}^{2 k+2} \sum_{i=0}^{\infty} \alpha_{i}^{2} \sum_{j_{1}=1}^{\infty} \beta_{i+j_{1}}^{2} \cdots \sum_{j_{k}=1}^{\infty} \beta_{i+j_{1}+\cdots+j_{k-1}+j_{k}}^{2} \\
& =\sum_{k=0}^{\infty} c_{Q}^{2 k+2} \sum_{0 \leq i<j_{1}<\cdots<j_{k}<\infty} \alpha_{i}^{2} \beta_{j_{1}}^{2} \cdots \beta_{j_{k}}^{2} \\
& \leq \sum_{k=0}^{\infty} c_{Q}^{2 k+2} A^{2} B_{1}^{2} \cdots B_{k}^{2},
\end{aligned}
$$


where $B_{k}^{2}:=\sum_{j=k}^{\infty} \beta_{j}^{2}$. Since $B^{2}<\infty$ entails $\lim _{k \rightarrow \infty} B_{k}^{2}=0$, for all $\varepsilon>0$, there exists $K \geq 1$ such that $B_{k}^{2}<\varepsilon / c_{Q}^{2}$ for all $k>K$. Hence,

$$
K_{Q} \leq c_{Q}^{2} A^{2}\left(\sum_{k=0}^{K}\left(c_{Q}^{2} B^{2}\right)^{k}+\sum_{k=K}^{\infty} \varepsilon^{k}\right)<\infty .
$$

Therefore, $A^{2}<\infty$ and $B^{2}<\infty$ imply that $K_{Q}<\infty$. The converse implication is obvious.

(ii) The proof follows from

$$
K_{Q}=\sum_{k=0}^{\infty} c_{Q}^{2 k+2} \sum_{i=0}^{\infty} \alpha_{i}^{2} \sum_{j_{1}=1}^{\infty} \beta_{j_{1}}^{2} \cdots \sum_{j_{k}=1}^{\infty} \beta_{j_{k}}^{2}=\sum_{k=0}^{\infty} c_{Q}^{2 k+2} A^{2}\left(B^{2}\right)^{k}=\frac{c_{Q}^{2} A^{2}}{1-c_{Q}^{2} B^{2}} .
$$

Remark 3. It is not difficult to show that conditions on the $\beta_{i, j}$ in Proposition 6(i) and (ii) are part of the following more general condition: $\limsup _{i \rightarrow \infty} \sum_{j=1}^{\infty} c_{Q}^{2} \beta_{i, j}^{2}<1$, which also guarantees that $K_{Q}<\infty$.

In the following proposition we obtain a sufficient condition for the existence of higher moments $\mathbb{E}\left|X_{t}\right|^{p}<\infty, p>2$, of the solution of projective equation (19). The proof of the proposition is based on a recurrent use of the Rosenthal-type inequality of Proposition 1, which contains an absolute constant $C_{p}$ depending only on $p$. For $p \geq 2$, define

$$
K_{Q, p}:=C_{p}^{2 / p} \sum_{i=0}^{\infty} \alpha_{i}^{2} \sum_{k=0}^{\infty}\left(c_{Q} C_{p}^{1 / p} \mu_{p}^{1 / p}\right)^{2 k+2} \sum_{j_{1}=1}^{\infty} \beta_{i, j_{1}}^{2} \cdots \sum_{j_{k}=1}^{\infty} \beta_{i+j_{1}+\cdots+j_{k-1}, j_{k}}^{2},
$$

where (recall) $\mu_{p}=\mathbb{E}\left|\zeta_{0}\right|^{p}$. Note that $C_{2}=\mu_{2}=1$; hence, $K_{Q, 2}=K_{Q}$ coincides with (23).

Proposition 7. Assume that the conditions of Theorem 1 hold and that $K_{Q, p}<\infty$ for some $p \geq 2$. Then $\mathbb{E}\left|X_{t}\right|^{p}<\infty$.

Proof. The proof is similar to that of Theorem 1(i). By Proposition 1,

$$
\begin{aligned}
\left(\mathbb{E}\left|X_{t}\right|^{p}\right)^{2 / p} & \leq C_{p}^{2 / p}\left(\left|\mathbb{E} X_{t}\right|^{p}+\mu_{p}\left(\sum_{s \leq t}\left(\mathbb{E}\left|g_{s, t}\right|^{p}\right)^{2 / p}\right)^{p / 2}\right)^{2 / p} \\
& =C_{p}^{2 / p} \mu_{p}^{2 / p} \sum_{s \leq t}\left(\mathbb{E}\left|g_{s, t}\right|^{p}\right)^{2 / p} .
\end{aligned}
$$

Using condition (22), Proposition 1, and the inequality $(a+b)^{q} \leq a^{q}+b^{q}, 0<q \leq 1$, we obtain the following recurrent inequality:

$$
\begin{aligned}
\left(\mathbb{E}\left|g_{s, t}\right|^{p}\right)^{2 / p} & \leq\left(c_{Q}^{p} \mathbb{E}\left|\alpha_{t-s}+\sum_{s<u \leq t} \beta_{t-u, u-s} \zeta_{u} g_{u, t}\right|^{p}\right)^{2 / p} \\
& \leq c_{Q}^{2} C_{p}^{2 / p}\left(\left|\alpha_{t-s}\right|^{p}+\mu_{p}\left(\sum_{s<u \leq t}\left(\left|\beta_{t-u, u-s}\right|^{p} \mathbb{E}\left|g_{u, t}\right|^{p}\right)^{2 / p}\right)^{p / 2}\right)^{2 / p} . \\
& \leq c_{Q}^{2} C_{p}^{2 / p}\left(\left|\alpha_{t-s}\right|^{2}+\mu_{p}^{2 / p} \sum_{s<u \leq t} \beta_{t-u, u-s}^{2}\left(\mathbb{E}\left|g_{u, t}\right|^{p}\right)^{2 / p}\right) .
\end{aligned}
$$

Iterating the last inequality as in the proof of Theorem 1 we obtain $\left(\mathbb{E}\left|X_{t}\right|^{p}\right)^{2 / p} \leq K_{Q, p}<\infty$, with $K_{Q, p}$ given in (29). 
Finally, let us consider the case in which $X_{t}$ of (19) satisfies the weak dependence condition in (14) for the invariance principle.

Proposition 8. Let $\left\{X_{t}\right\}$ satisfy the conditions of Theorem 1, and let $\Omega(2)$ be defined in (14). Then

$$
\Omega(2) \leq \sum_{i=0}^{\infty}\left|\alpha_{i}\right| \sum_{k=0}^{\infty} c_{Q}^{k+1} \sum_{j_{1}=1}^{\infty}\left|\beta_{i, j_{1}}\right| \cdots \sum_{j_{k}=1}^{\infty}\left|\beta_{i+j_{1}+\cdots+j_{k-1}, j_{k}}\right| .
$$

In particular, if the quantity on the right-hand side of $(30)$ is finite, $\left\{X_{t}\right\}$ satisfies the functional central limit theorem in (15).

Proof. The proof follows from (27) and the inequality $\left|\sum x_{i}\right|^{1 / 2} \leq \sum\left|x_{i}\right|^{1 / 2}$.

\section{Examples}

\subsection{Finitely dependent projective equations.}

Consider equation (19), where $\alpha_{i}=\beta_{i, j}=0$ for all $i>m$ and some $m \geq 0$. Since $Q(0)=0$, we have the corresponding equation

$$
X_{t}=\mu+\sum_{t-m<s \leq t} \zeta_{s} Q\left(\alpha_{t-s}+\sum_{s<u \leq t} \beta_{t-u, u-s}\left(\mathbb{E}_{[u, t]} X_{t}-\mathbb{E}_{[u+1, t]} X_{t}\right)\right) \text {, }
$$

where the right-hand side is $\mathcal{F}_{[t-m+1, t]}$-measurable. In particular, $\left\{X_{t}\right\}$ of (31) is an $m$ dependent process. We may ask if the above process can be represented as a moving average of length $m$ with respect to some i.i.d. innovations. In other words, we may ask if there exists an i.i.d. standardized sequence $\left\{\eta_{s}, s \in \mathbb{Z}\right\}$ and coefficients $c_{j}, 0 \leq j<m$, such that

$$
X_{t}=\sum_{t-m<s \leq t} c_{t-s} \eta_{s}, \quad t \in \mathbb{Z}
$$

To construct a negative counterexample to the above question, consider the simple case of (31) with $m=2, \mu=0, \alpha_{1}=0, \beta_{0,1}=1$, and $Q\left(\alpha_{0}\right)=1$ :

$$
X_{t}=\zeta_{t} Q\left(\alpha_{0}\right)+\zeta_{t-1} Q\left(\alpha_{1}+\beta_{0,1} \mathbb{E}_{[t, t]} X_{t}\right)=\zeta_{t}+\zeta_{t-1} Q\left(\zeta_{t}\right)
$$

Assume that $\mathbb{E} Q\left(\zeta_{t}\right)=0$. Then $\mathbb{E} X_{t} X_{t-1}=0$ and $\mathbb{E} X_{t}^{2}=1+\mathbb{E} Q^{2}\left(\zeta_{0}\right)$. On the other hand, from (32) with $m=2$ we obtain $0=\mathbb{E} X_{t} X_{t-1}=c_{0} c_{1}$, implying that $\left\{X_{t}\right\}$ is an i.i.d. sequence.

Let us show that the last conclusion contradicts the form of $X_{t}$ in (33) under general assumptions on $Q$ and the distribution of $\zeta=\zeta_{0}$. Assume that $\zeta$ is symmetric, $\infty>\mathbb{E} \zeta^{4}>$ $\left(\mathbb{E} \zeta^{2}\right)^{2}=1$, and that $Q$ is antisymmetric. Then

$$
\operatorname{cov}\left(X_{t}^{2}, X_{t-1}^{2}\right)=\mathbb{E} Q^{2}(\zeta)\left\{\left(\mathbb{E} \zeta^{4}-1\right)+\left(\mathbb{E} \zeta^{2} Q^{2}(\zeta)-\mathbb{E} Q^{2}(\zeta)\right)\right\}
$$

Assume, in addition, that $Q$ is monotone nondecreasing on $[0, \infty)$. Then $\mathbb{E} \zeta^{2} Q^{2}(\zeta) \geq$ $\mathbb{E} \zeta^{2} \mathbb{E} Q^{2}(\zeta)=\mathbb{E} Q^{2}(\zeta)$, implying that $\operatorname{cov}\left(X_{t}^{2}, X_{t-1}^{2}\right)>0$. As a consequence, (33) is not a moving average of length 2 in some standardized i.i.d. sequence. 


\subsection{Linear kernel $Q$.}

For the linear kernel $Q(x)=c_{Q} x$, the solution of (19) can be written explicitly as $X_{t}=$ $\mu+\sum_{k=1}^{\infty} X_{t}^{(k)}$, where $X_{t}^{(1)}=c_{Q} \sum_{i=0}^{\infty} \alpha_{i} \zeta_{t-i}$ is a linear process and

$$
X_{t}^{(k+1)}=c_{Q}^{k+1} \sum_{i=0}^{\infty} \alpha_{i} \sum_{j_{1}, \ldots, j_{k}=1}^{\infty} \beta_{i, j_{1}} \cdots \beta_{i+j_{1}+\cdots+j_{k-1}, j_{k}} \zeta_{t-i} \zeta_{t-i-j_{1}} \cdots \zeta_{t-j_{1}-\cdots-j_{k}}
$$

for $k \geq 1$ is a Volterra series of order $k+1$ (see [6, p. 22]), which are orthogonal in the sense that $\mathbb{E} X_{t}^{(k)} X_{s}^{(\ell)}=0, t, s \in \mathbb{Z}, k, \ell \geq 1, k \neq \ell$.

Let $H_{(-\infty, t]}^{2} \subset L_{(-\infty, t]}^{2}$ be the subspace spanned by products $1, \zeta_{s_{1}} \cdots \zeta_{s_{k}}, s_{1}<\cdots<s_{k} \leq$ $t, k \geq 1$. Clearly, the above Volterra series $X_{t}, X_{t}^{(k)} \in H_{(-\infty, t]}^{2}$ for all $t \in \mathbb{Z}$ (corresponding to linear $Q$ ) constitutes a very special class of projective processes. For example, the process in (33) cannot be expanded as such a series unless $Q$ is linear. To show the last fact, decompose (33) as $X_{t}=Y_{t}+Z_{t}$, where $Y_{t}:=\zeta_{t}+\alpha \zeta_{t-1} \zeta_{t} \in H_{(-\infty, t]}^{2}, \alpha:=\mathbb{E} \zeta Q(\alpha)$, and $Z_{t}:=$ $\zeta_{t-1}\left(Q\left(\zeta_{t}\right)-\alpha \zeta_{t}\right)$ is orthogonal to $H_{(-\infty, t]}^{2}, Z_{t} \neq 0$; hence, $X_{t} \notin H_{(-\infty, t]}^{2}$.

\subsection{The LARCH model.}

The linear ARCH (LARCH) model, introduced by Robinson [19], is defined by the equations

$$
r_{t}=\sigma_{t} \zeta_{t}, \quad \sigma_{t}=\alpha+\sum_{j=1}^{\infty} \beta_{j} r_{t-j}
$$

where $\left\{\zeta_{t}\right\}$ is a standardized i.i.d. sequence and the coefficients $\beta_{j}$ satisfy $B:=\left\{\sum_{j=1}^{\infty} \beta_{j}^{2}\right\}^{1 / 2}<$ $\infty$. The LARCH model was studied in [3], [9], [11], [12], [13], and other papers. In financial modeling, the $r_{t}$ are interpreted as (asset) returns and $\sigma_{t}$ as volatilities. Of particular interest is the case when the $\beta_{j}$ in (34) are proportional to ARFIMA coefficients, in which case it is possible to rigorously prove long memory of the volatility and the (squared) returns. It is well known [9] that a second-order strictly stationary solution $\left\{r_{t}\right\}$ to (34) exists if and only if

$$
B<1
$$

in which case it can be represented by the convergent orthogonal Volterra series

$$
r_{t}=\sigma_{t} \zeta_{t}, \quad \sigma_{t}=\alpha\left(1+\sum_{k=1}^{\infty} \sum_{j_{1}, \ldots, j_{k}=1}^{\infty} \beta_{j_{1}} \cdots \beta_{j_{k}} \zeta_{t-j_{1}} \cdots \zeta_{t-j_{1}-\cdots-j_{k}}\right)
$$

Clearly, the last series is a particular case of the Volterra series of the previous example. We conclude that, under condition (35), the volatility process $\left\{X_{t}=\sigma_{t}\right\}$ of the LARCH model satisfies the projective equation (21) with linear function $Q(x)=x$ and $\alpha_{j}=\alpha \beta_{j}$. Note that (35) coincides with the condition $c_{Q}^{2} B^{2}<1$ of Proposition 6(ii) for the existence of a solution to (21).

From Proposition 7, the following new result about the existence of higher-order moments of the LARCH model is derived.

Corollary 2. Assume that

$$
C_{p}^{1 / p} \mu_{p}^{1 / p} B<1
$$



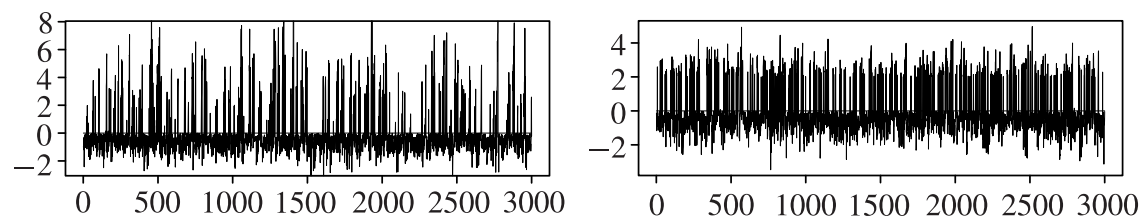

Figure 1: Trajectories of solutions of (39) for $p=10$. Left: $Q(x)=\mathbf{1}(x>0)$. Right: $Q(x)=\mathbf{1}(0<$ $x<2$ ).

where $\mu_{p}=\mathbb{E}\left|\zeta_{0}\right|^{p}$ and $C_{p}$ is the absolute constant from Proposition $1, p \geq 2$. Then $\mathbb{E}\left|r_{t}\right|^{p}=$ $\mu_{p} \mathbb{E}\left|\sigma_{t}\right|^{p}<\infty$. Moreover,

$$
\mathbb{E}\left|\sigma_{t}\right|^{p} \leq \frac{\alpha^{2} C_{p}^{4 / p} \mu_{p}^{2 / p} B^{2}}{1-C_{p}^{2 / p} \mu_{p}^{2 / p} B^{2}} .
$$

Proof. The proof follows from Proposition 7 and the easy fact that, for the LARCH model, $K_{Q, p}$ of (29) coincides with the right-hand side of (37).

Condition (36) can be compared with the sufficient condition for $\mathbb{E}\left|r_{t}\right|^{p}<\infty, p=2,4, \ldots$, in [12, Lemma 3.1]:

$$
\left(2^{p}-p-1\right)^{1 / 2} \mu_{p}^{1 / p} B<1 .
$$

Although the best constant $C_{p}$ in Rosenthal's inequality is not known, (36) seems much weaker than (38), especially when $p$ is large. See, e.g. [15], where it is shown that $C_{p}^{1 / p}=O(p / \log p)$ as $p \rightarrow \infty$.

\subsection{Projective 'threshold' equations.}

Consider the projective equation

$$
X_{t}=\zeta_{t}+\sum_{j=1}^{p} \zeta_{t-j} Q\left(\mathbb{E}_{[t-j+1, t]} X_{t}\right)
$$

where $1 \leq p<\infty$ and $Q$ is a bounded measurable function with $Q(0)=1$. If $Q$ is a step function, $Q(x)=\sum_{k=1}^{q} c_{k} \mathbf{1}\left(x \in I_{k}\right)$, where $\bigcup_{k=1}^{q} I_{k}=\mathbb{R}$ is a partition of $\mathbb{R}$ into disjoint intervals $I_{k}, 1 \leq k \leq q$, the process in (39) follows different 'moving average regimes' in the regions $\mathbb{E}_{[t-j+1, t]} X_{t} \in I_{k}, 1 \leq j \leq p$, exhibiting a 'projective threshold effect'. See Figure 1, in which we plot a trajectory with a single threshold at $x=0$ in the left-hand graph, and a trajectory with two threshold points at $x=0$ and $x=2$ in the right-hand graph.

\section{Simulations}

Solutions of projective equations can be easily simulated using a truncated expansion $X_{t}^{(M)}=$ $\sum_{t-M \leq s \leq t} g_{s, t} \zeta_{s}$ instead of the infinite series in (5). We chose the truncation level $M$ equal to the sample size $M=n=3000$ in the subsequent simulations. The coefficients $g_{s, t}$ of the projective equations are computed very fast from recurrent formula (25) and simulated values $\zeta_{s},-M \leq s \leq n$. The innovations were taken standard normal. For better comparisons, we used the same sequence $\zeta_{s},-M \leq s \leq n$, in all simulations.

The stationary solution of (21) was simulated for three different choices of $Q$ and two choices of the coefficients $\alpha_{j}$ and $\beta_{j}$. The first choice of coefficients was $\alpha_{j}=0.5^{j}$ and 
$\beta_{j}=c 0.9^{j}$, corresponding to a short memory process $\left\{X_{t}\right\}$. The second choice was $\alpha_{j}=$ $\Gamma(d+j) / \Gamma(d) \Gamma(j+1)$ and $\beta_{j}=c \alpha_{j}$ with $d=0.4$, corresponding to a long memory process $\left\{X_{t}\right\}$ with coefficients as in $\operatorname{ARFIMA}(0, d, 0)$. The value of $c>0$ was chosen so that $c_{Q}^{2} B^{2}=0.9<1$. The latter condition guarantees the existence of a stationary solution to $(21)$; see Proposition 6.

The simulated trajectories and (smoothed) histograms of marginal densities strongly depend on the kernel $Q$. We used the following functions:

$$
Q_{1}(x)=x, \quad Q_{2}(x)=\max (0, x), \quad Q_{3}(x)= \begin{cases}x, & x \in[0,1], \\ 2-x, & x \in[1,2] \\ 0, & \text { otherwise }\end{cases}
$$

Clearly, $Q_{i}, i=1,2,3$, in (40) satisfy (22) with $c_{Q}=1$ and the Lipschitz condition (48) below. Note that $Q_{3}$ is bounded and supported in the compact interval [0,2], while $Q_{1}$ and $Q_{2}$ are unbounded, the latter being bounded from below. Also, note that, for $\beta_{j} \equiv 0$ and the choice of $\alpha_{j}$ above, the projective process $\left\{X_{t}\right\}$ of (21) agrees with $\operatorname{AR}(0.5)$ for $\alpha_{j}=0.5^{j}$ and with $\operatorname{ARFIMA}(0,0.4,0)$ for $\alpha_{j}=\Gamma(d+j) / \Gamma(d) \Gamma(j+1)$ in all three cases in (40)

A general impression from our simulations is that in all cases of $Q$ in (40), the coefficients $\alpha_{j}$ account for the persistence and $\beta_{j}$ for the clustering of the process. We observe that as the $\beta_{j}$ values increase, the process becomes more asymmetric and its empirical density diverges from the normal density (plotted as dashed lines in Figures 2-4 with parameters equal to the empirical mean and variance of the simulated series). In the case of unbounded $Q=Q_{1}, Q_{2}$ and long memory ARFIMA coefficients, the marginal distribution seems strongly skewed to the left with a very light left tail and a much heavier right tail. On the other hand, in the case of geometric coefficients, the density for $Q=Q_{1}, Q_{2}$ seems rather symmetric although heavy tailed. The case $Q=Q_{3}$ corresponding to bounded $Q$ seems to result in an asymmetric distribution with light tails.
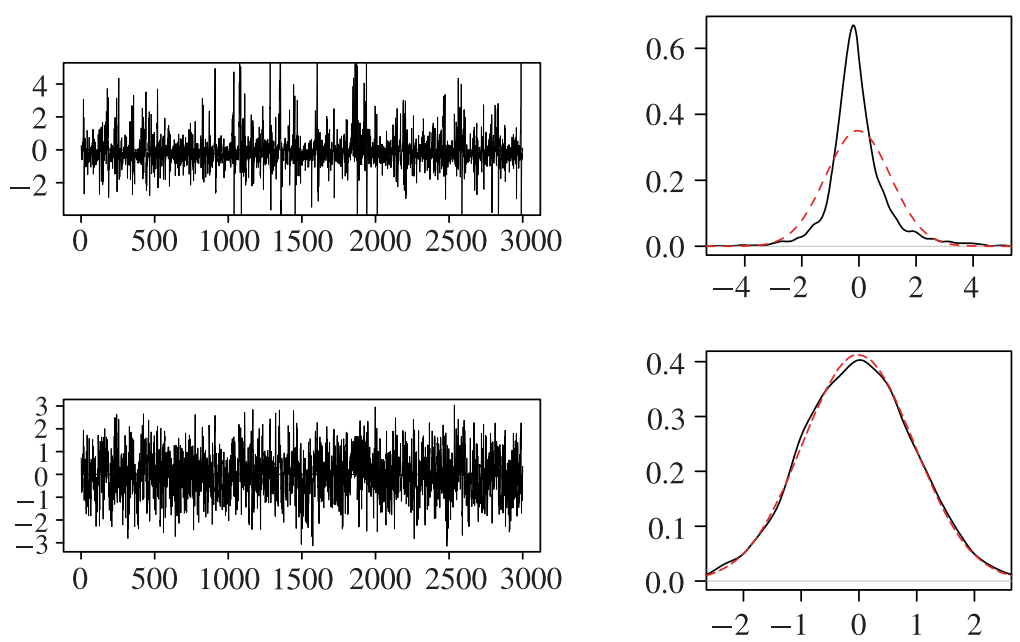

Figure 2: Trajectories and (smoothed) histograms of solutions to projective equation (21) with $Q(x)=$ $Q_{1}(x)=x$. Top: $\alpha_{j}=(0.5)^{j}$ and $\beta_{j}=c(0.9)^{j}$. Bottom: $\alpha_{j}=(0.5)^{j}$ and $\beta_{j}=0$. 

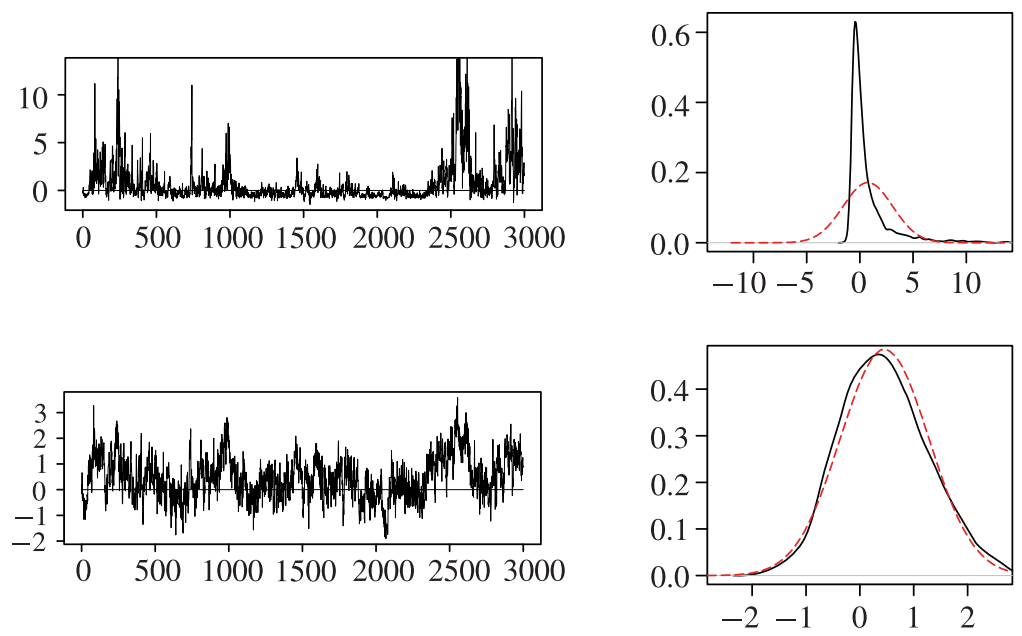

FIGURE 3: Trajectories and (smoothed) histograms of solutions to projective equation (21) with $Q(x)=$ $Q_{2}(x)=\max (x, 0) . T o p: \alpha_{j}=\operatorname{ARFIMA}(0,0.4,0)$ and $\beta_{j}=c \alpha_{j} . \operatorname{Bottom}: \alpha_{j}=\operatorname{ARFIMA}(0,0.4,0)$ and $\beta_{j}=0$.
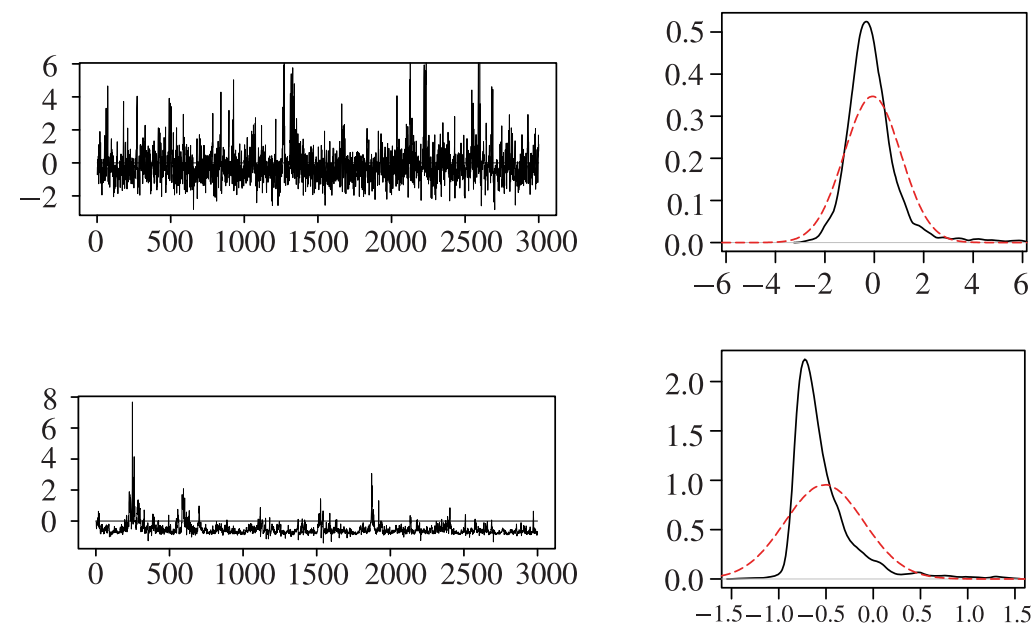

FIGURE 4: Trajectories and (smoothed) histograms of solutions to projective equation (21) with $Q(x)=$ $Q_{3}(x)=$ the 'triangle function' in (40). Top: $\alpha_{j}=(0.5)^{j}$ and $\beta_{j}=c(0.9)^{j}$. Bottom: $\alpha_{j}=$ $\operatorname{ARFIMA}(0,0.4,0)$ and $\beta_{j}=c \alpha_{j}$

\section{Modifications}

Equation (19) can be modified in several ways. The first modification is obtained by taking the $\alpha_{t-s}$ 'outside of $Q$, i.e.

$$
X_{t}=\mu+\sum_{s \leq t} \zeta_{s} \alpha_{t-s} Q\left(\sum_{s<u \leq t} \beta_{t-u, u-s}\left(\mathbb{E}_{[u, t]} X_{t}-\mathbb{E}_{[u+1, t]} X_{t}\right)\right),
$$

where $\alpha_{i}, \beta_{i, j}$, and $Q$ satisfy similar conditions as in (19). However, note that (22) implies that $Q(0)=0$ in which case (41) has the trivial solution $X_{t} \equiv \mu$. To avoid the last eventuality, 
condition (22) must be changed. Instead, we assume that $Q$ is a measurable function satisfying

$$
Q(x)^{2} \leq c_{0}^{2}+c_{1}^{2} x^{2}, \quad x \in \mathbb{R},
$$

for some $c_{0}, c_{1} \geq 0$. Define

$$
\tilde{K}_{Q}:=c_{0}^{2} \sum_{k=0}^{\infty} c_{1}^{2 k} \sum_{i=0}^{\infty} \alpha_{i}^{2} \sum_{j_{1}=1}^{\infty} \alpha_{i+j_{1}}^{2} \beta_{i, j_{1}}^{2} \cdots \sum_{j_{k}=1}^{\infty} \alpha_{i+j_{1}+\cdots+j_{k}}^{2} \beta_{i+j_{1}+\cdots+j_{k-1}, j_{k}}^{2} .
$$

Proposition 9 below can be proved similarly to Theorem 1 and its proof is thus omitted.

Proposition 9. (i) Assume that condition (42) holds and that

$$
\tilde{K}_{Q}<\infty \text {. }
$$

Then there exists a unique solution $\left\{X_{t}\right\}$ of $(41)$, which is written as a projective moving average of the form (10) with coefficients $g_{t-k, t}$ recursively defined as

$$
g_{t-k, t}:=\alpha_{k} Q\left(\sum_{i=0}^{k-1} \beta_{i, k-i} \zeta_{t-i} g_{t-i, t}\right), \quad k=1,2, \ldots, \quad g_{t, t}:=\alpha_{0} Q(0) .
$$

(ii) In the case of the linear function $Q(x)=c_{0}+c_{1} x$, condition (43) is also necessary for the existence of a solution to (41).

Remark 4. Let $A_{k}^{2}:=\sum_{i=k}^{\infty} \alpha_{i}^{2}$ and $\left|\beta_{i, j}\right| \leq \bar{\beta}$. Then

$$
\tilde{K}_{Q} \leq c_{0}^{2} \sum_{k=0}^{\infty}\left(c_{1} \bar{\beta}\right)^{2 k} \sum_{i=0}^{\infty} \alpha_{i}^{2} \sum_{j_{1}=1}^{\infty} \alpha_{i+j_{1}}^{2} \cdots \sum_{j_{k}=1}^{\infty} \alpha_{i+j_{1}+\cdots+j_{k}}^{2} \leq c_{0}^{2} \sum_{k=0}^{\infty}\left(c_{1} \bar{\beta}\right)^{2 k} A_{0}^{2} A_{1}^{2} \cdots A_{k}^{2} .
$$

Hence, $A^{2}=A_{0}^{2}<\infty$ and $\bar{\beta}<\infty$ imply that $\tilde{K}_{Q}<\infty$ for any $c_{0}, c_{1}$, and $\bar{\beta}$; see the proof of Proposition 6.

Projective stochastic equations (19) and (41) can be further modified by including projections of lagged variables. Consider the following extension of (19):

$$
X_{t}=\mu+\sum_{s \leq t} \zeta_{s} Q\left(\alpha_{t-s}+\sum_{u=s+1}^{t-1} \beta_{t-1-u, u-s}\left(\mathbb{E}_{[u, t-1]} X_{t-1}-\mathbb{E}_{[u+1, t-1]}\right) X_{t-1}\right) .
$$

Here $\alpha_{i}, \beta_{i, j}$, and $Q$ are the same as in (19) and the only new feature is that $t$ is replaced by $t-1$ in the inner sum on the right-hand side of the equation. This fact allows us to study nonstationary solutions of (44) with a given projective initial condition $X_{t}=X_{t}^{0}, t \leq 0$, and the convergence of $X_{t}$ to the equilibrium as $t \rightarrow \infty$; however, we will not pursue this topic in the present paper. The following proposition is a simple extension of Theorem 1 and its proof is thus omitted.

Proposition 10. Let $\alpha_{i}, \beta_{i, j}$, and $Q$ satisfy the conditions of Theorem 1, including (22) and(24). Then there exists a unique solution $\left\{X_{t}\right\}$ of (44), which is written as a projective moving average of the form (10) with coefficients $g_{t-k, t}$ recursively defined as $g_{t-k, t}:=Q\left(\alpha_{k}\right), k=0,1$, and

$$
g_{t-k, t}:=Q\left(\alpha_{k}+\sum_{i=0}^{k-2} \beta_{i, k-1-i} \zeta_{t-1-i} g_{t-1-i, t-1}\right), \quad k \geq 2 .
$$


Finally, consider projective equation (16) with $\mu_{t} \equiv 0$ and kernels $Q_{s, t}=Q_{t-s}\left(x_{s+1, t-1}\right.$, $\ldots, x_{s+1, s}$ ) depending on $t-s$ real variables, where $Q_{0}=1$ and

$$
Q_{j}\left(x_{1}, \ldots, x_{j}\right)=\frac{d\left(x_{1}\right)}{1} \frac{d\left(x_{2}\right)+1}{2} \frac{d\left(x_{3}\right)+2}{3} \cdots \frac{d\left(x_{j}\right)+j-1}{j}, \quad j \geq 1,
$$

where $d(x), x \in \mathbb{R}$, is a measurable function taking values in the interval $\left(-\frac{1}{2}, \frac{1}{2}\right)$. More explicitly,

$$
X_{t}=\sum_{j=0}^{\infty} Q_{j}\left(\mathbb{E}_{[t-j+1, t-1]} X_{t-1}, \mathbb{E}_{[t-j+1, t-2]} X_{t-2}, \ldots, \mathbb{E}_{[t-j+1, t-j]} X_{t-j}\right) \zeta_{t-j},
$$

where $\mathbb{E}_{[t-j+1, t-j]} X_{t-j}=\mathbb{E} X_{t}=0$. Note that, when $d(x)=d$ is constant, $\left\{X_{t}\right\}$ in (46) is a stationary $\operatorname{ARFIMA}(0, d, 0)$ process. Time-varying fractionally integrated processes with deterministic coefficients of the form (45) were studied in [17] and [18]. We expect that (46) features a 'random' memory intensity depending on the values of the process. A rigorous study of the long memory properties of this model does not seem easy. On the other hand, solvability of (46) can be established similarly to the previous cases (see below).

Proposition 11. Let $d(x)$ be a measurable function taking values in $\left(-\frac{1}{2}, \frac{1}{2}\right)$ and such that $\sup _{x \in \mathbb{R}} d(x) \leq \bar{d}$, where $\bar{d} \in\left(0, \frac{1}{2}\right)$. Then there exists a unique stationary solution $\left\{X_{t}\right\}$ of (46), which is written as a projective moving average of the form (10) with coefficients $g_{s, t}$ recursively defined as $g_{t, t}:=1$ and

$$
g_{s, t}:=Q_{t-s}\left(\sum_{s<u \leq t-1} \zeta_{u} g_{u, t-1}, \sum_{s<u \leq t-2} \zeta_{u} g_{u, t-2}, \ldots, 0\right), \quad s<t,
$$

with $Q_{t-s}$ defined at (45).

Proof. Note that $\sup _{x_{1}, \ldots, x_{j} \in \mathbb{R}}\left|Q_{j}\left(x_{1}, \ldots, x_{j}\right)\right| \leq \Gamma(\bar{d}+j) / \Gamma(\bar{d}) \Gamma(j)=: \psi_{j}$ and $\sum_{j=0}^{\infty} \psi_{j}^{2}<\infty$. Therefore, the $g_{s, t}$ in (47) satisfy $\sum_{s \leq t} \mathbb{E} g_{s, t}^{2}<\infty$ for any $t \in \mathbb{Z}$. The rest of the proof is analogous as the case of Theorem 1 .

\section{Long memory}

In this section we study the long memory properties (the decay of covariance and partial sums limits) of projective equations (19) and (41) in the case when the coefficients $\alpha_{j}$ decay slowly as $j^{d-1}, 0<d<\frac{1}{2}$.

Theorem 2. Let $\left\{X_{t}\right\}$ be the solution of projective equation (19) satisfying the conditions of Theorem 1, and let $\mu=\mathbb{E} X_{t}=0$. Assume, in addition, that $Q$ is a Lipschitz function, namely, there exists a constant $c_{L}>0$ such that

$$
|Q(x)-Q(y)|<c_{L}|x-y|, \quad x, y \in \mathbb{R},
$$

and that there exist $\kappa>0$ and $0<d<\frac{1}{2}$ such that

$$
b_{j}:=Q\left(\alpha_{j}\right) \sim \kappa j^{d-1} \text { as } j \rightarrow \infty
$$

and

$$
\bar{\beta}_{j}:=\max _{0 \leq i<j}\left|\beta_{i, j-i}\right|=o\left(b_{j}\right) \quad \text { as } j \rightarrow \infty
$$


Then, as $t \rightarrow \infty$,

$$
\mathbb{E} X_{0} X_{t} \sim \sum_{k=0}^{\infty} b_{k} b_{t+k} \sim \kappa_{d}^{2} t^{2 d-1},
$$

where $\kappa_{d}^{2}:=\kappa^{2} B(d, 1-d)$ and $B(d, 1-d)$ is the beta function. Moreover, as $n \rightarrow \infty$,

$$
n^{-1 / 2-d} \sum_{t=1}^{[n \tau]} X_{t} \rightarrow{ }_{D[0,1]} c_{\kappa, d} B_{H}(\tau),
$$

where $B_{H}$ is a fractional Brownian motion with parameter $H=d+\frac{1}{2}$ and variance $\mathbb{E} B_{H}^{2}(t)=$ $t^{2 H}$, and $c_{\kappa, d}^{2}:=\kappa^{2} B(d, 1-d) / d(1+2 d)$.

Proof. Let us note that statements (50) and (51) are well known when $\beta_{i, j} \equiv 0$, in which case $X_{t}$ coincides with the linear process $Y_{t}:=\sum_{s \leq t} b_{t-s} \zeta_{s}$. See, e.g. [10, Proposition 3.2.1 and Corollary 4.4.1].

The natural idea of the proof is to approximate $\left\{X_{t}\right\}$ by the linear process $\left\{Y_{t}\right\}$. For $t \geq 0$ and $k \geq 0$, define

$$
\begin{gathered}
r_{t}^{X}:=\mathbb{E} X_{0} X_{t}=\sum_{s \leq 0} \mathbb{E}\left[g_{s, 0} g_{s, t}\right], \quad r_{t}^{Y}:=\mathbb{E} Y_{0} Y_{t}=\sum_{s \leq 0} b_{-s} b_{t-s}, \\
\phi_{t-k, t}:=g_{t-k, t}-b_{k}=Q\left(\alpha_{k}+\sum_{i=0}^{k-1} \beta_{i, k-i} \zeta_{t-i} g_{t-i, t}\right)-Q\left(\alpha_{k}\right)
\end{gathered}
$$

Then

$$
\begin{aligned}
r_{t}^{X}-r_{t}^{Y} & =\sum_{s \leq 0} \mathbb{E}\left[\left(b_{-s}+\phi_{s, 0}\right)\left(b_{t-s}+\phi_{s, t}\right)-b_{-s} b_{t-s}\right] \\
& =\sum_{s \leq 0} b_{-s} \mathbb{E}\left[\phi_{s, t}\right]+\sum_{s \leq 0} b_{t-s} \mathbb{E}\left[\phi_{s, 0}\right]+\sum_{s \leq 0} \mathbb{E}\left[\phi_{s, 0} \phi_{s, t}\right] \\
& =: \sum_{i=1}^{3} \rho_{i, t} .
\end{aligned}
$$

Using (48), we obtain

$$
\begin{aligned}
\left|\mathbb{E} \phi_{t-k, t}\right|^{2} & \leq \mathbb{E} \phi_{t-k, t}^{2} \\
& \leq c_{L}^{2} \mathbb{E}\left(\sum_{i=0}^{k-1} \beta_{i, k-i} \zeta_{t-i} g_{t-i, t}\right)^{2} \\
& =c_{L}^{2}\left(\sum_{i=0}^{k-1} \beta_{i, k-i}^{2} \mathbb{E} g_{t-i, t}^{2}\right) \\
& \leq \bar{\beta}_{k}^{2} c_{L}^{2}\left(\sum_{i=0}^{\infty} \mathbb{E} g_{t-i, t}^{2}\right) \\
& \leq \bar{\beta}_{k}^{2} c_{L}^{2} K_{Q} .
\end{aligned}
$$

This and condition (49) imply that

$$
\left|\mathbb{E} \phi_{t-k, t}\right|+\mathbb{E}^{1 / 2} \phi_{t-k, t}^{2} \leq \delta_{k} k^{d-1} \quad \text { for all } t, k \geq 0,
$$


where $\delta_{k} \rightarrow 0(k \rightarrow \infty)$. Therefore, for any $t \geq 1$,

$$
\begin{aligned}
& \left|\rho_{1, t}\right| \leq C \sum_{k=1}^{\infty} k^{d-1}(t+k)^{d-1} \delta_{t+k} \leq C \delta_{t}^{\prime} t^{2 d-1}, \\
& \left|\rho_{2, t}\right| \leq C \sum_{k=1}^{\infty} k^{d-1} \delta_{k}(t+k)^{d-1} \leq C \delta_{t}^{\prime} t^{2 d-1}, \\
& \left|\rho_{3, t}\right| \leq \sum_{s \leq t} \mathbb{E}^{1 / 2}\left[\phi_{s, 0}^{2}\right] \mathbb{E}^{1 / 2}\left[\phi_{s, t}^{2}\right] \leq C \sum_{k=1}^{\infty} k^{d-1}(t+k)^{d-1} \delta_{k} \delta_{t+k} \leq C \delta_{t}^{\prime} t^{2 d-1},
\end{aligned}
$$

where $\delta_{k}^{\prime} \rightarrow 0(k \rightarrow \infty)$. This proves $(50)$.

To show (51), consider $Z_{t}:=X_{t}-Y_{t}=\sum_{u \leq t} \phi_{u, t} \zeta_{u}, t \in \mathbb{Z}$. By the stationarity of $\left\{Z_{t}\right\}$, for any $s \leq t$, we have $\operatorname{cov}\left(Z_{t}, Z_{s}\right)=\sum_{u \leq 0} \mathbb{E}\left[\phi_{u, 0} \phi_{u, t-s}\right] \leq \sum_{u \leq 0} \mathbb{E}^{1 / 2}\left[\phi_{u, 0}^{2}\right] \mathbb{E}^{1 / 2}\left[\phi_{u, t-s}^{2}\right]=$ $o\left((t-s)^{2 d-1}\right)$ (see above) and, therefore, $\mathbb{E}\left(\sum_{t=1}^{n} Z_{t}\right)^{2}=o\left(n^{2 d+1}\right)$, implying that

$$
n^{-d-(1 / 2)} \sum_{t=1}^{[n \tau]} X_{t}=n^{-d-1 / 2} \sum_{t=1}^{[n \tau]} Y_{t}+o_{p}(1) \text {. }
$$

Therefore, partial sums of $\left\{X_{t}\right\}$ and $\left\{Y_{t}\right\}$ tend to the same limit $c_{\kappa, d} B_{H}(\tau)$, in the sense of weak convergence of finite-dimensional distributions. The tightness in $D[0,1]$ follows from (50) and the Kolmogorov criterion. This completes the proof.

A similar but somewhat different approximation by a linear process applies in the case of projective equations of (41). Let us discuss a special case of $\beta_{i, j}$ :

$$
\beta_{i, j}=1 \quad \text { for all } i=0,1, \ldots \text { and } j=1,2, \ldots
$$

Note that, for such $\beta_{i, j}, \sum_{s<u<t} \beta_{t-u, u-s}\left(\mathbb{E}_{[u, t]}-\mathbb{E}_{[u+1, t]}\right) X_{t}=\mathbb{E}_{[s+1, t]} X_{t}, s<t$, and the corresponding projective equation (41) with $\mu=0$ and $\alpha_{i}=b_{i}$ coincides with (9). Recall that, for bounded $\beta_{i, j}$ as in (52), condition (42) on $Q$ together with $\sum_{i=0}^{\infty} \alpha_{i}^{2}<\infty$ guarantees the existence of the stationary solution $\left\{X_{t}\right\}$ (see Remark 4). We will also need the following additional condition:

$$
\mathbb{E}\left(Q\left(\mathbb{E}_{[s, 0]} X_{0}\right)-Q\left(X_{0}\right)\right)^{2} \rightarrow 0 \quad \text { as } s \rightarrow-\infty .
$$

Since $\mathbb{E}\left(\mathbb{E}_{[s, 0]} X_{0}-X_{0}\right)^{2} \rightarrow 0$ as $s \rightarrow-\infty$, (53) is satisfied if $Q$ is Lipschitz; otherwise, conditions (53) and (42) allow $Q$ to be discontinuous. Define

$$
c_{Q, d}^{2}:=\left(\mathbb{E}\left[Q\left(X_{0}\right)\right]\right)^{2} B(d, 1-d) .
$$

Theorem 3. Let $\left\{X_{t}\right\}$ be the solution of projective equation (41) with $\mu=0, \beta_{i, j}$ as in (52), $Q$ satisfying (42), and

$$
\alpha_{k} \sim k^{d-1} \text { as } k \rightarrow \infty \text { for } 0<d<\frac{1}{2} .
$$

In addition, let (53) hold. Then

$$
\mathbb{E} X_{0} X_{t} \sim c_{Q, d}^{2} t^{2 d-1} \text { as } t \rightarrow \infty
$$

and

$$
n^{-1 / 2-d} \sum_{t=1}^{[n \tau]} X_{t} \rightarrow_{D[0,1]} c_{Q, d}^{\prime} B_{H}(\tau), \quad c_{Q, d}^{\prime}:=\frac{c_{Q, d}}{(d(1+2 d))^{1 / 2}}
$$


Proof. Similarly as in the proof of the previous theorem, let $Y_{t}:=\sum_{s \leq t} b_{t-s} \zeta_{s}, b_{k}:=$ $\alpha_{k} \mathbb{E}\left[Q\left(X_{0}\right)\right], r_{t}^{X}:=\mathbb{E} X_{0} X_{t}$, and $r_{t}^{Y}:=\mathbb{E} Y_{0} Y_{t}, t \geq 0$. Relation (55) follows from

$$
r_{t}^{X}-r_{t}^{Y}=o\left(t^{2 d-1}\right) \quad \text { as } t \rightarrow \infty
$$

We have $X_{t}=\sum_{s \leq t} g_{s, t} \zeta_{s}, g_{s, t}=\alpha_{t-s} Q\left(\mathbb{E}_{[s+1, t]} X_{t}\right), \mathbb{E} X_{t}^{2}=\sum_{s \leq t} \mathbb{E} g_{s, t}^{2}<\infty$, and $\mathbb{E}\left[Q\left(\mathbb{E}_{[s+1, t]} X_{t}\right)^{2}\right] \leq c_{0}^{2}+c_{1}^{2} \mathbb{E}\left(\mathbb{E}_{[s+1, t]} X_{t}\right)^{2} \leq c_{0}^{2}+c_{1}^{2} \mathbb{E} X_{t}^{2}<C$. Decompose $r_{t}^{X}=r_{1, t}^{X}+r_{2, t}^{X}$, where

$$
r_{1, t}^{X}:=\sum_{s \leq 0} \alpha_{s} \alpha_{t+s} \mathbb{E}\left[Q\left(\mathbb{E}_{[s+1,0]} X_{0}\right)\right] \mathbb{E}\left[Q\left(\mathbb{E}_{[1, t]} X_{t}\right)\right], \quad r_{2, t}^{X}:=\sum_{s \leq 0} \alpha_{s} \alpha_{t+s} \gamma_{s, t},
$$

and where

$$
\left|\gamma_{s, t}\right|:=\left|\mathbb{E}\left[Q\left(\mathbb{E}_{[s+1,0]} X_{0}\right)\left\{Q\left(\mathbb{E}_{[s+1, t]} X_{t}\right)-Q\left(\mathbb{E}_{[1, t]} X_{t}\right)\right\}\right]\right| \leq \tilde{\gamma}_{1, s}^{1 / 2} \tilde{\gamma}_{2, s, t}^{1 / 2} .
$$

Here $\tilde{\gamma}_{1, s}:=\mathbb{E}\left[Q^{2}\left(\mathbb{E}_{[s+1,0]} X_{0}\right)\right] \leq C$ (see above), while

$$
\begin{aligned}
\left|\tilde{\gamma}_{2, s, t}\right| & :=\mathbb{E}\left[\left(Q\left(\mathbb{E}_{[s+1, t]} X_{t}\right)-Q\left(\mathbb{E}_{[1, t]} X_{t}\right)\right)^{2}\right] \\
& =\mathbb{E}\left[\left(Q\left(\mathbb{E}_{[s+1-t, 0]} X_{0}\right)-Q\left(\mathbb{E}_{[1-t, 0]} X_{0}\right)\right)^{2}\right] \\
& \rightarrow 0 \quad \text { as } t \rightarrow \infty,
\end{aligned}
$$

uniformly in $s \leq 0$, according to (53). Hence, from (54), it follows that

$$
\left|r_{2, t}^{X}\right|=o\left(t^{2 d-1}\right) \quad \text { as } t \rightarrow \infty .
$$

Accordingly, it suffices to prove (57) with $r_{t}^{X}$ replaced by $r_{1, t}^{X}$. We have

$$
r_{1, t}^{X}=r_{t}^{Y}+\sum_{s \leq 0} \alpha_{s} \alpha_{t+s} \phi_{1, s, t}+\sum_{s \leq 0} \alpha_{s} \alpha_{t+s} \phi_{2, s, t}+\sum_{s \leq 0} \alpha_{s} \alpha_{t+s} \phi_{3, s, t},
$$

where the 'remainders'

$$
\begin{aligned}
\phi_{1, s, t} & :=\mathbb{E}\left[Q\left(X_{0}\right)\right]\left\{\mathbb{E}\left[Q\left(\mathbb{E}_{[s+1,0]} X_{0}\right)\right]-\mathbb{E}\left[Q\left(X_{0}\right)\right]\right\}, \\
\phi_{2, s, t} & :=\mathbb{E}\left[Q\left(X_{0}\right)\right]\left\{\mathbb{E}\left[Q\left(\mathbb{E}_{[1-t, 0]} X_{0}\right)\right]-\mathbb{E}\left[Q\left(X_{0}\right)\right]\right\}, \\
\text { and } \quad \phi_{3, s, t} & :=\left(\mathbb{E}\left[Q\left(\mathbb{E}_{[s+1,0]} X_{0}\right)\right]-\mathbb{E}\left[Q\left(X_{0}\right)\right]\right)\left(\mathbb{E}\left[Q\left(\mathbb{E}_{[1-t, 0]} X_{0}\right)\right]-\mathbb{E}\left[Q\left(X_{0}\right)\right]\right)
\end{aligned}
$$

can be estimated similarly to (58), leading to the asymptotics in (59) for each of the three sums in the above decomposition of $r_{1, t}^{X}$. This proves (55).

Let us prove (56). Consider the convergence of one-dimensional distributions for $\tau=1$, namely,

where $S_{n}^{X}:=\sum_{t=1}^{n} X_{t}$. Then (60) follows from

$$
n^{-d-1 / 2} S_{n}^{X} \rightarrow \mathcal{N}\left(0, \sigma^{2}\right), \quad \sigma=c_{Q, d}^{\prime},
$$

$$
\mathbb{E}\left(S_{n}^{X}-S_{n}^{Y}\right)^{2}=o\left(n^{1+2 d}\right),
$$

where $S_{n}^{Y}:=\sum_{t=1}^{n} Y_{t}$ and $Y_{t}$ is as above. We have

$$
\begin{aligned}
\mathbb{E}\left(S_{n}^{X}-S_{n}^{Y}\right)^{2} & =\mathbb{E}\left(\sum_{s \leq n} \zeta_{s} \sum_{t=1 \vee s}^{n} \alpha_{t-s} \tilde{Q}_{s, t}\right)^{2} \\
& =\sum_{s \leq n} \sum_{t_{1}, t_{2}=1 \vee s}^{n} \alpha_{t_{1}-s} \alpha_{t_{2}-s} \mathbb{E}\left[\tilde{Q}_{s, t_{1}} \tilde{Q}_{s, t_{2}}\right],
\end{aligned}
$$


where $\tilde{Q}_{s, t}:=Q\left(\mathbb{E}_{[s+1, t]} X_{t}\right)-\mathbb{E}\left[Q\left(X_{0}\right)\right]$. Let us prove that, uniformly in $s \leq t_{1}$,

$$
\mathbb{E}\left[\tilde{Q}_{s, t_{1}} \tilde{Q}_{s, t_{2}}\right]=o(1) \quad \text { as } t_{2}-t_{1} \rightarrow \infty .
$$

We have, for $s \leq t_{1} \leq t_{2}$,

$$
\begin{aligned}
\mathbb{E}\left[\tilde{Q}_{s, t_{1}} \tilde{Q}_{s, t_{2}}\right]= & \mathbb{E}\left[\tilde{Q}_{s, t_{1}}\left\{Q\left(\mathbb{E}_{\left[s+1, t_{2}\right]} X_{t_{2}}\right)-\mathbb{E}\left[Q\left(X_{0}\right)\right]\right\}\right] \\
= & \mathbb{E}\left[\tilde{Q}_{s, t_{1}}\right]\left\{\mathbb{E}\left[Q\left(\mathbb{E}_{\left[t_{1}+1, t_{2}\right]} X_{t_{2}}\right)\right]-\mathbb{E}\left[Q\left(X_{0}\right)\right]\right\} \\
& +\mathbb{E}\left[\tilde{Q}_{s, t_{1}}\left\{Q\left(\mathbb{E}_{\left[s+1, t_{2}\right]} X_{t_{2}}\right)-Q\left(\mathbb{E}_{\left[t_{1}+1, t_{2}\right]} X_{t_{2}}\right)\right\}\right] \\
= & : \psi_{s, t_{1}, t_{2}}^{\prime}+\psi_{s, t_{1}, t_{2}}^{\prime \prime},
\end{aligned}
$$

where we have used the fact that $\tilde{Q}_{s, t_{1}}$ and $Q\left(\mathbb{E}_{\left[t_{1}+1, t_{2}\right]} X_{t_{2}}\right)$ are independent. Here, thanks to (53), we see that

$$
\begin{aligned}
\left|\psi_{s, t_{1}, t_{2}}^{\prime}\right| & \leq \mathbb{E}^{1 / 2}\left[\tilde{Q}_{s, t_{1}}^{2}\right] \mathbb{E}^{1 / 2}\left[\left\{Q\left(\mathbb{E}_{\left[t_{1}-t_{2}+1,0\right]} X_{0}\right)-Q\left(X_{0}\right)\right\}^{2}\right] \\
& \leq C \mathbb{E}^{1 / 2}\left[\left\{Q\left(\mathbb{E}_{\left[t_{1}-t_{2}+1,0\right]} X_{0}\right)-Q\left(X_{0}\right)\right\}^{2}\right] \\
& \rightarrow 0
\end{aligned}
$$

uniformly in $s \leq t_{1} \leq t_{2}$ as $t_{2}-t_{1} \rightarrow \infty$. The same is true for $\left|\psi_{s, t_{1}, t_{2}}^{\prime \prime}\right|$ since it is completely analogous to (58). This proves (63). Next, with (62) in mind, split $\mathbb{E}\left(S_{n}^{X}-S_{n}^{Y}\right)^{2}=: T_{n}=$ $T_{1, n}+T_{2, n}$, where

$$
T_{1, n}:=\sum_{s \leq n} \sum_{t_{1}, t_{2}=1 \vee s}^{n} \mathbf{1}\left(\left|t_{1}-t_{2}\right|>K\right) \ldots, \quad T_{2, n}:=\sum_{s \leq n} \sum_{t_{1}, t_{2}=1 \vee s}^{n} \mathbf{1}\left(\left|t_{1}-t_{2}\right| \leq K\right) \ldots,
$$

where $K$ is a large number. By (63), for any $\varepsilon>0$, we can find $K>0$ such that

$$
\sup _{\left\{s \leq t_{1}<t_{2}: t_{2}-t_{1}>K\right\}}\left|\mathbb{E}\left[\tilde{Q}_{s, t_{1}} \tilde{Q}_{s, t_{2}}\right]\right|<\varepsilon
$$

and, therefore,

$$
\left|T_{1, n}\right|<\varepsilon \sum_{s \leq n} \sum_{t_{1}, t_{2}=1 \vee s}^{n}\left|\alpha_{t_{1}-s} \alpha_{t_{2}-s}\right| \leq C \varepsilon \sum_{t_{1}, t_{2}=1}^{n}\left|\bar{r}_{t_{1}-t_{2}}\right| \leq C \varepsilon n^{1+2 d}
$$

holds for all large enough $n>1$, where $\bar{r}_{t}:=\sum_{i=0}^{\infty}\left|\alpha_{i} \alpha_{t+i}\right|=O\left(t^{2 d-1}\right)$ in view of (54). On the other hand, $\left|T_{2, n}\right| \leq C K n=o\left(n^{1+2 d}\right)$ for any fixed $K<\infty$. Then (61) follows, implying the finite-dimensional convergence in (56). The tightness in (56) follows from (55) and the Kolmogorov criterion, similarly as in the proof of Theorem 2. This completes the proof.

Remark 5. Shao and $\mathrm{Wu}$ [25] discussed partial sums limits of fractionally integrated nonlinear processes $Y_{t}=(1-L)^{-d} u_{t}, t \in \mathbb{Z}$, where $L X_{t}=X_{t-1}$ is the backward shift, $(1-L)^{d}=$ $\sum_{j=0}^{\infty} \psi_{j}(d) L^{j}, d \in(-1,1)$, is the fractional differentiation operator, and $\left\{u_{t}\right\}$ is a causal Bernoulli shift, i.e.

$$
u_{t}=F\left(\ldots, \zeta_{t-1}, \zeta_{t}\right), \quad t \in \mathbb{Z},
$$

in i.i.d. random variables $\left\{\zeta_{t}, t \in \mathbb{Z}\right\}$. The weak dependence condition on $\left\{u_{t}\right\}$ in (64), analogous to (14) and guaranteeing the weak convergence of normalized partial sums of $\left\{Y_{t}\right\}$ towards a fractional Brownian motion, is written in terms of projections $P_{0} u_{t}=\left(\mathbb{E}_{[0, t]}-\mathbb{E}_{[1, t]}\right) u_{t}$ :

$$
\Omega(q):=\sum_{t=1}^{\infty}\left\|P_{0} u_{t}\right\|_{q}<\infty
$$


Here $\|\xi\|_{q}:=\mathbb{E}^{1 / q}|\xi|^{q}$ and $q=2$ for $0<d<\frac{1}{2}$; see [25, Theorem 2.1], and also [23] and [24]. The above-mentioned papers verify (65) for several classes of Bernoulli shifts. It is of interest to verify (65) for projective moving averages. For $X_{t}$ of (5) and $0<d<\frac{1}{2}$, $u_{t}:=(1-L)^{d} X_{t}=\sum_{s \leq t} \zeta_{s} G_{s, t}$ is a well-defined projective moving average with coefficients

$$
G_{s, t}:=\sum_{s \leq v \leq t} \psi_{t-v}(d) g_{s, v}, \quad s \leq t ;
$$

see Proposition 2. For concreteness, let $g_{s, t}=\psi_{t-s}(-d) Q\left(\mathbb{E}_{[s+1, t]} X_{t}\right)$ as in Theorem 3 with $\alpha_{j}=\psi_{j}(-d)$. We have $\Omega(2)=\sum_{t=1}^{\infty}\left\|G_{0, t}\right\|_{2}$, where

$$
\left\|G_{0, t}\right\|_{2}^{2}=\mathbb{E}\left[\sum_{v=0}^{t} \psi_{t-v}(d) \psi_{v}(-d) Q\left(\mathbb{E}_{[1, v]} X_{v}\right)\right]^{2}=\mathbb{E}\left[\sum_{v=0}^{t-1} \psi_{t-v}(d) \psi_{v}(-d) Q_{v, t}\right]^{2},
$$

where $Q_{v, t}:=Q\left(\mathbb{E}_{[1, v]} X_{v}\right)-Q\left(\mathbb{E}_{[1, t]} X_{t}\right)$ and we used $\sum_{v=0}^{t} \psi_{t-v}(d) \psi_{v}(-d)=0, t \geq 1$, in the last equality. Note that $\psi_{t-v}(d) \psi_{v}(-d)<0$ have the same sign and $Q_{v, t} \approx Q\left(X_{v}\right)-Q\left(X_{t}\right)$ are not negligible in (66). Therefore, we conjecture that

$$
\left\|G_{0, t}\right\|_{2}^{2}=O\left(\sum_{v=0}^{t-1}\left|\psi_{t-v}(d) \psi_{v}(-d)\right|\right)^{2}=O\left(t^{-2(1-d)}\right)
$$

and, hence, $\Omega(2)=\infty$ for $0<d<\frac{1}{2}$. The above argument suggests that projective moving averages posses a different 'memory mechanism' from the fractionally integrated processes in $[25]$.

\section{Acknowledgements}

The authors thank an anonymous referee for useful remarks. This work was supported by the Research Council of Lithuania, under grant number MIP-063/2013.

\section{References}

[1] Baillie, R. T. and Kapetanios, G. (2008). Nonlinear models for strongly dependent processes with financial applications. J. Econometrics 147, 60-71.

[2] Beran, J. (1994). Statistics for Long-Memory Processes (Monogr. Statist. Appl. Prob. 61). Chapman and Hall, New York.

[3] Berkes, I. And Horváth, L. (2003). Asymptotic results for long memory LARCH sequences. Ann. Appl. Prob. 13, 641-668.

[4] Davydov, Yu. A. (1970). The invariance principle for stationary process. Theory Prob. Appl. 15, 487-498.

[5] Dedecker, J. and Merlevède, F. (2003). The conditional central limit theorem in Hilbert spaces. Stoch. Process. Appl. 108, 229-262.

[6] Dedecker, J. et al. (2007). Weak Dependence (Lecture Notes Statist. 190). Springer, New York.

[7] Doukhan, P., Lang, G. And Surgailis, D. (2012). A class of Bernoulli shifts with long memory: asymptotics of the partial sums process. Preprint. University of Cergy-Pontoise.

[8] Doukhan, P., Oppenheim, G. And Taqqu, M. (eds) (2003). Theory and Applications of Long-Range Dependence. Birkhäuser, Boston, MA.

[9] Giraitis, L. AND Surgailis, D. (2002). ARCH-type bilinear models with double long memory. Stoch. Process. Appl. 100, 275-300.

[10] Giraitis, L., Koul, H. L. And Surgailis, D. (2012). Large Sample Inference for Long Memory Processes. Imperial College Press, London.

[11] Giraitis, L., Leipus, R. AND Surgailis, D. (2009). ARCH( $\infty)$ models and long memory properties. In Handbook of Financial Time Series, eds T. Mikosch et al., Springer, Berlin, pp. 71-84. 
[12] Giraitis, L., Robinson, P. M. And Surgailis, D. (2000). A model for long memory conditional heteroskedasticity. Ann. Appl. Prob. 10, 1002-1024.

[13] Giraitis, L., Leipus, R., Robinson, P. M. and Surgailis, D. (2004). LARCH, leverage, and long memory. J. Financial Econometrics 2, 177-210.

[14] Hall, P. And Heyde, C. C. (1980). Martingale Limit Theory and Application. Academic Press, New York.

[15] Hitczenko, P. (1990). Best constants in martingale version of Rosenthal's inequality. Ann. Prob. 18, 1656-1668.

[16] Ho, H.-C. And Hsing, T. (1997). Limit theorems for functionals of moving averages. Ann. Prob. 25, 1636-1669.

[17] Philippe, A., Surgailis, D. And Viano, M.-C. (2006). Invariance principle for a class of non stationary processes with long memory. C. R. Math. Acad. Sci. Paris 342, 269-274.

[18] Philippe, A., Surgailis, D. and Viano, M.-C. (2008). Time-varying fractionally integrated processes with nonstationary long memory. Theory Prob. Appl. 52, 651-673.

[19] Robinson, P. M. (1991). Testing for strong serial correlation and dynamic conditional heteroskedasticity in multiple regression. J. Econometrics 47, 67-84.

[20] Robinson, P. M. (2001). The memory of stochastic volatility models. J. Econometrics 101, 195-218.

[21] Stout, W. F. (1974). Almost Sure Convergence. Academic Press, New York.

[22] TAQQU, M. S. (1979). Convergence of integrated processes of arbitrary Hermite rank. Z. Wahrscheinlichkeitsth. 50, 53-83.

[23] Wu, W. B. (2005). Nonlinear system theory: another look at dependence. Proc. Nat. Acad. Sci. USA 102, 14150-14154.

[24] Wu, W. B. And Min, W. (2005). On linear processes with dependent innovations. Stoch. Process. Appl. 115, 939-958.

[25] Wu, W. B. ANd Shao, X. (2006). Invariance principles for fractionally integrated nonlinear processes. In Recent Developments in Nonparametric Inference and Probability (IMS Lecture Notes Monogr. Ser. 50), Institute of Mathematical Statistics, Beachwood, OH, pp. 20-30. 